\title{
ALGUNAS REFLEXIONES SOBRE LA PRÁCTICA PÚBLICA Y LA INTEGRACIÓN DE LAS PSICOTERAPIAS
}

\author{
ALBERTO FERNÁNDEZ LIRIA \\ Universidad de Alcalá \\ (Recibido el 10 de diciembre de 1998)
}

\begin{abstract}
La historia de la psicoterapia ha estado jalonada por la necesidad de demostrar, primero, su eficacia como procedimiento de tratamiento de los trastornos mentales y, segundo, de resolver el dilema creado por la imposibilidad de demostrar diferencias consistentes entre las intervenciones basadas en diferentes concepciones teóricas. En este artículo se revisan los intentos de resolver una y otra necesidad, y se argumenta la utilidad de las concepciones propias de las propuestas de las tres corrientes del movimiento ecléctico-integrador (eclecticismo técnico, integración teórica y factores comunes) para los psicoterapeutas con práctica en el sector público, que trabajan sin posibilidad de seleccionar pacientes.
\end{abstract}

Palabras clave: Psicoterapia, integración de las psicoterapias, psicoterapia en el sector público.

\section{Some reflections about psychotherapy in the public sector and the integration of psychotherapies}

In the history of psychotherapy we can find, firstly, the need to demonstrate its general effectiviness, and secondly, the need to solve the dilemma created by the impossibility to find differences in effectiveness among the interventions based upon different theoretical conceptions. In this paper the attempts to satisfy both needs are reviewed. Moreover, the utility of the ideas from the three versions of the movement for eclecticism and integration.of psychotherapies (technical electicism, theoretical integration and common factors) are emphasized in order to be used by the psychotherapists working in the public sector, bearing in mind that these psychoterapists do not have the possibility of selecting their patients.

Key words: Psychotherapy, psychotherapy integration, psychotherapy in the mental health system.

\section{INTRODUCCIÓN}

Hace unos años que me piden con cierta frecuencia que hable o escriba sobre psicoterapias en el sector público. Yo acepto con gusto hablar sobre ese tema porque es lo que hago y me gusta. Pero cada vez me cuesta más pensar por qué llaman así a la cosa de la que a mí me gusta hablar. No sé si la confusión tiene que ver con lo que los solicitantes puedan entender por psicoterapia. El uso común de ese término -l que aparece en los folletos que reparten a la salida del metro o se incluyen en las secciones de anuncios por palabrasincluye prácticas absolutamente diversas entre sí (unas se hacen tumbado, en otras se grita o se llora, a otras hay que ir con chandall o se combinan con masaje...), que además responden a propósitos completamente diferentes (el autoconocimiento, el crecimiento personal, la armonía con la naturaleza, la curación del cáncer, la espiritualidad). Esto no tiene en principio que preocuparnos. Los folletos que se reparten a la salida del metro y los

En este escrito se basó la comunicación del autor a la mesa sobre Psicoterapia en el sistema público, que tuvo lugar el 13 de diciembre de 1998 en Getxo, durante las XV Jornadas de la Asociación Española de Neuropsiquiatría. 
anuncios por palabras no son nuestros libros de texto. Y los neurólogos no dudan de su identidad profesional porque la gente hable de estar delicada o mal de los nervios, o atribuya sus dolencias a que estos objetos privilegiados del conocimiento científico - los nervios- se les hayan "agarrado» a la cabeza o al estómago por haberse duchado teniendo la regla. Pero pone de manifiesto que hemos de empezar precisando a qué nos referimos cuando hablamos de psicoterapia.

Hace unos años nos reunimos un grupo de psicoterapeutas que trabajábamos - y trabajamos- en el sector público en Madrid, convocados para el congreso de la Asociación Española de Neuropsicología (AEN), para elaborar una ponencia sobre las consecuencias que tenía el trabajo en el sector público sobre la integración de las psicoterapias (Fernández Liria, Hernández Monsalve y Rodríguez Vega, 1997). Consensuar algunos términos y conceptos, y acordar una definición de psicoterapia nos llevó varias sesiones. La formulamos así:

Psicoterapia es "un proceso de comunicación interpersonal entre un profesional experto (terapeuta) y un sujeto necesitado de ayuda por problemas de salud mental (paciente) que tiene como objeto producir cambios para mejorar la salud mental del segundo».

Añadíamos a renglón seguido que esta definición pretende circunscribirse a aquellas prácticas de las que reclaman el nombre de psicoterapias en las que se pretende actuar sobre problemas de salud mental (a punto estuvimos de utilizar el término trastornos mentales), excluyendo las que persiguen objetivos como el autoconocimiento, el desarrollo personal o el acceso a experiencias más o menos interesantes (que nos parecían irrelevantes para una reflexión planteada desde la psiquiatría pública).
Se matizó esta definición con algunas aclaraciones que cito textualmente:

Esta definición excluye otra serie de prácticas que pudieran, en alguna medi$d a$, ser consideradas afines, como son:

* Otras relaciones de ayuda (amigos...) en los que el que la da no es un profesional experto.

* Consejo médico o psicológico en el que el experto se limita a poner a disposición del cliente información que le permite realizar una elección cuya realización no está impedida por patología mental ${ }^{1}$.

* Manejo clínico de un tratamiento medicamentoso, en el que el objetivo es garantizar las condiciones (de información, confianza en el médico, expectativas, adherencia al tratamiento) que permiten optimizar el efecto de la medicación prescrita.

\section{Como nota adicional convinimos que:}

La psicoterapia supone la posibilidad de construir una versión del problema presentado por el paciente en el que éste aparece como implicado personalmente en el origen, el mantenimiento o las posibilidades de resolución del mismo (Fernández Liria y Rodríguez Vega, 1996).

Se trata, para lo que aquí nos interesa, de una definición que sitúa la psicoterapia entre los procedimientos de tratamiento de los trastornos mentales, es decir, de aquellas para las que la desinencia terapia no es tan sólo una metáfora. Con una definición así la locución en el sector público se refiere a una circuns-

\footnotetext{
${ }^{1}$ Es consejo si se dice algo como «debe dejar de fumar porque en su caso - bronquitis crónicafumar entraña tales y cuales riesgosn. Hay psicoterapia si se inicia una intervención para resolver el problema de que el cliente no puede dejar de fumar aunque conozca los peligros y quiera hacerlo. Lo mismo será aplicable en el caso de un consejero matrimonial.
} 
tancia de lugar (como sería psicoterapia en Cuenca, en el desierto o en la Luna...), y no de modo (como sería si habláramos de psicoterapia breve, cognitiva o de inspiración psicoanalítica).

Trabajar en el sector privado nos exonera de la obligación de atener nuestra práctica a esta definición (podemos aceptar un cliente nada más que porque tiene deseo de pagar para que le escuchemos), y de la obligación de proporcionar al paciente la alternativa mas eficiente y no la que él ha elegido pedirnos en el mercado de las psicoterapias. En esto -y, en principio, sólo en esto- consiste la mayor libertad que el sector privado ofrece para la práctica de la psicoterapia. Pero lo que aquí diremos es de aplicación para cualquier práctica que se ajuste a nuestra definición, aunque se realice en el sector privado. Desde luego el lugar impone sus condiciones. En el grupo que antes citaba señalamos algunas que se recogen en el Cuadro 1.

Suponemos que por un acuerdo implícito con esta idea - del que la AEN es al menos parcialmente responsable- la psicoterapia individual y de grupo se encuentran entre las prestaciones supuestamente ofertadas por el Sistema Nacional de Salud. La psicoterapia individual, familiar o de grupo, sola o en combinación con fármacos, aparece como definición de un $73 \%$ de los actos asistenciales llevados a cabo sobre la población adulta en los Centros de Salud Mental de la Comunidad de Madrid en los que existe un registro acumulativo de casos psiquiátricos. Para la población infanto-juvenil este porcentaje se eleva hasta el $92 \%$ (Fernández Liria, Hernández Monsalve, Rodríguez Vega, Benito Cano y Mas Hesse 1997).

Cuando mostramos estos datos frecuentemente oímos comentarios como: «Bueno: vamos a ver a qué llamamos psicoterapia; en realidad en el sector público psicoterapias de verdad apenas hacemos». Son psicoterapias de verdad, deduzco, las que se realizan en condiciones que no se dan en el sector público (sesiones estrictamente semanales o aún mas frecuentes, objetivos de modificación profunda de la estructura de la personalidad, ¿pago directo?...). En realidad sabemos que estas psicoterapias de verdad tampoco se dan en el sector privado, cuyos clientes, hasta donde han podido averiguar quienes se han tomado la molestia de investigarlo, reciben en su inmensa mayoría menos de seis sesiones (Garfield 1994). Nosotros, manteniendo la actitud que adoptamos en aquél grupo de consenso, vamos a adoptar la postura inversa a la implícita en esa afirmación. La psicoterapia de verdad es la que se lleva a cabo en esa práctica que se realiza, realmente, en los centros. ¿Cómo llamar si no a lo que hacen nuestros psicólogos, a lo que hago yo con los muchos pacientes a los que nunca he recetado un fármaco, o con aquellos a los que, aunque se lo receto, consumen mucho más tiempo de consulta del necesario para garantizar la prescripción correcta y la información necesaria para hacer buen uso del mismo?

Si nuestra idea, nuestras teorías sobre la psicoterapia, no se ajusta a nuestra

Cuadro 1. La psicoterapia en el sector público

- Sin selección de pacientes (el valor de la indicación terapéutica)

- Consideración de la eficiencia (y eficiencia relativa)

- Son condiciones diferentes no necesariamente peores

- No es una adaptación o versión para pobres

- La pregunta NO es

¿Sobre qué pacientes puedo actuar de un modo más eficaz con esta técnica concreta? SI NO ¿Con qué técnica puedo obtener mejores -o menos malos-resultados sobre este paciente concreto? 
práctica, lo que debemos cambiar es nuestra idea: debemos dotarnos de teorías capaces de ayudarnos a seleccionar nuestras actuaciones, explicar por qué funcionan cuando funcionan, y de mejorarlas o diseñar otras nuevas cuando no funcionan o lo hacen de un modo insuficiente.

Hoy tenemos suficientes datos provenientes de la investigación para guiar esta tarea de construcción teórica y para desarrollar nuevos y fundamentados programas de investigación. Voy a intentar resumirlos brevemente, aún a riesgo de caer a veces casi en la caricatura. Y lo voy a hacer refiriéndome a algunos hitos de la historia de la investigación en psicoterapia que, aunque parezca extraño, han tenido en ambas ocasiones, la forma de veredictos.

\section{EL VEREDICTO DE EYSENCK Y LA CUESTIÓN DE LA EFECTIVIDAD DE LA PSICOTERAPIA}

El primero es un veredicto emitido por un investigador tan riguroso como Eysenck y viene a poner de manifiesto que, en el momento de su publicación (1952), la psicoterapia era una práctica extendida y prestigiada por cuyo liderazgo competían diversas escuelas pero no existían pruebas serias de que fuera eficaz. En realidad Eysenck concluía algo aún peor: que la evidencia existente apuntaba a que la psicoterapia era menos eficaz que la permanencia en lista de espera y que, de algún modo, impedía la remisión espontánea de los trastornos neuróticos. Pero el fundamento de esta conclusión fue pronta y justamente discutido junto con la metodología del estudio que dio lugar a él. Una de las reacciones ante este primer veredicto fue la puesta en marcha de una cantidad importante de investigación y el desarrollo de una metodología investigadora en oca- siones finísima (Fernández Liria 1993, 1994; Lambert y Bergin, 1994). A estas alturas de siglo la efectividad general de las psicoterapias, tanto frente a la ausencia de tratamiento como frente a placebo, nos parece fuera de duda. Lambert y Bergin (1994) revisaron los resultados de los estudios meta-analíticos realizados sobre el efecto de la psicoterapia sobre diversos trastornos poniendo de manifiesto que su magnitud de efecto es semejante a la de otros tratamientos utilizados en medicina. La utilización de meta-análisis en la investigación sobre psicoterapias ha venido a resolver alguna de las dificultades de este tipo de investigación como, por ejemplo, la exigüidad del tamaño de las muestras, o la introducción de sesgos debidos a características de los investigadores o de los lugares en los que se lleva a cabo la investigación.

Desde luego que la efectividad demostrada no es para sentirse satisfecho. Pero no lo es para ninguno de los modos de tratamiento de que disponemos en psiquiatría. Un editorial reciente del Current Opinion in Psychiatry (Rush, 1996), una publicación poco sospechosa de veleidades antipsiquiátricas, nos recordaba que, aunque la tasa de respuesta de los trastornos depresivos a los fármacos antidepresivos estaba en torno al $50 \%$ (lo que tampoco es ninguna maravilla), respuesta (disminución estadísticamente significativa de la puntuación, por ejemplo, en la escala de Hamiltón) no es sinónimo de remisión. La mayor parte de los trabajos sobre efectividad de los psicofármacos no proporcionan datos sobre la tasa de remisión y si la extraemos de los escasísimos que sí los proporcionan, dicha tasa se sitúa en torno al $25 \%$. Si consideramos que la remisión sintomática suele preceder a la recuperación del funcionamiento previo en las esferas laboral, social y familiar, la perspectiva es aún peor. Este es el panorama general en que nos movemos en la terapéutica de los tratamientos 
en general. Un panorama que nos obliga a ser modestos (aunque el marketing y la modestia se den de bofetadas y la mayor parte de la información que nos llega a los psiquiatras lo haga en forma de instrumento de marketing de la industria farmacéutica). Debemos ser modestos, por tanto, a la hora de apreciar la efectividad de la psicoterapia, pero no más que para valorar la de cualquier otro medio de tratamiento de los trastornos mentales.

Si el sistema público de atención a la salud debe proporcionar los instrumentos disponibles para la recuperación de la salud de los ciudadanos que la han perdido, la psicoterapia se encuentra entre ellos con la misma legitimidad que la cirugía o la antibioterapia.

\section{¿CUÁNDO, PARA QUIÉN, Y QUÉ PSICOTERAPIA? EL VEREDICTO DEL PÁJARO DODO}

Si la investigación realizada desde la formulación del veredicto de Eysenck hasta 1980 y en la década posterior, tuvo éxito en la demostración de la efectividad que tenían las psicoterapias (en términos generales) para el tratamiento de los trastornos mentales en general, y de algunos cuadros clínicos en particular, no fue capaz de satisfacer lo que constituía uno de los estímulos más importantes para muchos investigadores adscritos a diferentes escuelas de psicoterapia: demostrar la superioridad de las intervenciones basadas en una determinada teoría (que podría así ser considerada como más correcta o más acertada que el resto para explicar los trastornos mentales y su tratamiento), en comparación con las basadas en las demás.

Esta incapacidad dio lugar a la formulación del segundo de los jalones a los que hacíamos referencia, marcado por lo que Lester Luborski (Luborski, Singer y Luborski 1975) bautizó como veredicto del pájaro Dodo , y que consiste en la imposibilidad del abultado trabajo de investigación realizado hasta la fecha para demostrar diferencias consistentes entre intervenciones psicoterapéuticas basadas en diferentes teorías o escuelas, imposibilidad a la que se ha añadido la dificultad encontrada para definir un placebo en psicoterapia y la comprobación de la magnitud de efecto lograda por alguno de los placebos propuestos. Este segundo veredicto ha abierto dos líneas de trabajo que parten de supuestos distintos y persiguen objetivos diferentes, aunque se miran entre sí con creciente interés. Por un lado el intento de buscar formas incontaminadas de cada una de las terapias propuestas, procurando la acción de los factores que cada una considera más específicos. Se trata en esta línea de garantizar la pureza de las intervenciones aplicadas y estudiar la especificidad de los factores terapéuticos que activan. En su versión extrema ha dado lugar a todo el proceso de manualización de las psicoterapias. En el campo de la investigación la manualización ha permitido avances notables y ha dado lugar a anécdotas interesantes como la del descubrimiento por parte de Fairburn de la terapia interpersonal de la bulimia nerviosa cuando estaba tratando de formalizar un placebo para demostrar la eficacia de la terapia cognitivo-conductual que él mismo practicaba (Fairburn 1993; Fairburn, Jones, Pevler, Carr, Solomon, O’Connor, Burton y Hope, 1991)

Respecto a lo que aquí nos interesa, la manualización, ha tenido ya una repercusión importante sobre sistemas de dispensación de servicios muy volcados en la consideración de los costes, como el norteamericano y, sin duda, lo tendrá en el nuestro. En torno a ella se ha producido recientemente un movimiento que

\footnotetext{
${ }^{2}$ Personaje de Alicia en el pais de las maravillas en cuya boca pone Lewis Carroll la frase "todos han ganado y cada cual tiene derecho a su premion.
} 
puede marcar un tercer jalón, también con forma de veredicto, en la historia reciente de las psicoterapias: el de la creación, por parte de la Asociación Americana de Psicólogos (APA) de un catálogo de tratamientos empíricamente validados, que incluye sólo a aquellos que, debidamente manualizados y contrastados vía ensayo clínico randomizado y doble ciego, han demostrado ser eficaces frente a un trastorno también debidamente definido de forma operativa con criterios DSM o similar. La APA se ha apresurado a afirmar que con este catálogo no pretende crear un émulo de la Food and Drug Admistration para los tratamientos psicológicos. Pero en cualquier caso ha destapado un especie de caja de Pandora a partir de la cual ya hay compañías de seguros que pretenden pagar sólo el número y tipo de sesiones establecidas en los manuales según el tipo de trastorno, y terapeutas discutiendo con vehemencia sobre si este movimiento les priva de la libertad y creatividad necesarias para actuar como tales en un entorno como el de la clínica cotidiana, que poco tiene que ver con el creado artificialmente para llevar a cabo las investigaciones en las que se han fundamentado tales manuales (por cierto que alguien con interés en ello debería periódicamente traducir e importar el debate al terreno equivalente de la efectividad de los psicofármacos donde, aunque la industria no tenga interés en hacerlo demasiado patente, sucede exactamente lo mismo). Creo que no tardaremos en encontrar gestores que pretenderán discutir en estos términos los catálogos de prestaciones de nuestros servicios públicos.

$\mathrm{Al}$ margen de estas consideraciones, los manuales representan un instrumento útil para la investigación y, en ocasiones, para la formación de psicoterapeutas. Alguno de ellos ha sido específicamente adaptado para las condiciones de trabajo en nuestro sector público y ha dado lugar a una cierta cantidad de docencia e investigación en ese campo (Diéguez Porres, Morales Hevia y González Suárez,1997; Fernández Liria, Rodríguez Vega, Diéguez Porres, González Suárez y Morales Hevia, 1997)

La segunda línea de trabajo abierta por el veredicto del pájaro Dodo es la que ha dado origen a lo que se conoce como el movimiento por el eclecticismo o la integración en psicoterapia, cuyas características han sido resumidas por Norcross y Arkowitz (1992). En una encuesta realizada entre los profesionales del sector público de la zona Sur de Madrid con motivo de una ponencia de la AEN (Desviat, Fernández Liria y Mas Hesse 1996) se puso de manifiesto que el $76 \%$ declaraba que fundamentaba su práctica en más de una orientación teórica y que el 93\% utilizaba o prescribía técnicas basadas en diferentes orientaciones teóricas. Hoy esta tendencia ha encontrado un cauce de expresión y discusión organizada a través de asociaciones como la Society for the Exploration of Psychotherapy Integration o revistas como el Journal of Psychotherapy Integration, $y$ ha dado lugar a toda una revolución en el modo de entender la práctica de la psicoterapia.

En el trabajo al que antes hacíamos referencia, Norcross y Arkowitz (1992) plantean tres modos de responder, desde una perspectiva ecléctico-integradora, al dilema del pájaro Dodo, que creemos tienen su traducción en tendencias y prácticas que detectamos en el sector público.

El primero de ellos la bautizaron como eclecticismo técnico y ha encontrado sus mejores representantes en Lazarus y Beutler. Según los partidarios de esta tendencia los avances en la práctica clínica no tienen porqué corresponderse - ni se corresponden de hecho- con los de nuestras teorías sobre la naturaleza de los trastornos mentales. Por eso, aunque hoy tenemos información suficiente para 
saber que determinadas modalidades de intervención basadas en distintas teorías, son útiles ante diferentes situaciones, no estamos en condiciones de dotarnos de una teoría capaz de explicar a la vez por qué funciona cada uno de ellos. Lo que estos autores sostienen es la posibilidad de construir sistemas capaces de permitirnos seleccionar intervenciones de un modo sistemático (y no intuitivamente) para los problemas que presenta cada paciente (o como acertadamente propone Beutler, para los problemas que se le presentan a cada pareja terapeuta-paciente). Tanto Lazarus (1992) como Beutler, se han negado a definirse como integradores y creen que la única postura posible es el eclecticismo. Ello no significa que los terapeutas que lo practican deban carecer de postura térica para entender los trastornos mentales. De hecho Beutler personalmente participa de una concepción basada en la teoría de la persuasión, y Lazarus es un conductista con concepciones deudoras de Wolpe y de Albert Bandura. Pero tanto el eclecticismo sistemático de Beutler (1983) como la terapia multimodal de Lazarus (1981), se presentan como sistemas que pueden permitir diseñar intervenciones, independientemente de cuál sea la posición teórica del terapeuta que las indica (por eso, y no por otra cosa, son eclécticos). El eclecticismo intuitivo es casi una necesidad en un marco que, como el del sector público, impide seleccionar pacientes. Lo que estos autores han abierto es una vía para pensar qué y cómo estamos haciendo lo que ya hacemos, y para mostrarnos hasta qué punto algunos de los instrumentos que estamos intentando utilizar para hacerlo (como el DSM o la CIE) están inmensamente alejados de contemplar los elementos que podrían orientarnos a la hora de tomar decisiones para confeccionar un plan terapéutico.

La segunda respuesta al dilema del pájaro Dodo es la propuesta, en amigable oposición a los anteriores, por los partidarios de la integración teórica. El desafío de los partidarios de esta postura es construir teorías capaces de dar cuenta $a$ la vez de la capacidad de producir efectos terapéuticos, demostrada por las intervenciones basadas en escuelas diferentes. Son precursores tempranos de esta postura Alexander (1963) y Dollard y Miller (1950). Más recientemente Wachtel $(1977,1987,1993,1997)$ ha propuesto un modelo original de psicoterapia que sintetiza principios y hallazgos del psicoanálisis, la modificación de conducta y las terapias sistémicas. Horowitz (Horowitz 1988, 1997; Horowitz, Marmar y Krupnick, 1984) ha construido un modelo teórico y un método práctico que reformula conceptos psicoanalíticos en términos de psicología cognitiva y emplea conceptos basados en la teoría de los roles y el análisis transaccional, y Ryle (1990, 1995a, 1995b) ha propuesto un modelo de psicoterapia cognitivo-analítica, que pretende adscribir a una teoría del desarrollo basada en Vigotsky. Estos autores nos enseñan a no ignorar, a efectos de nuestras reflexiones, los hallazgos de los compañeros de otras escuelas y de quienes hacen psicología en los medios académicos y nos animan a no abandonar el intento de construir teorías que nos permitan comprender cada elemento de nuestra práctica (en lugar de, por ejemplo, atribuir lo que no cuadra con nuestros prejuicios al hecho de estar trabajando en el sector público, en unas condiciones que no son las artificialmente propuestas desde prácticas que sólo son sostenibles a base de seleccionar los pacientes que responden a ellas).

La última de las posibles respuestas al dilema del pájaro Dodo es la planteada por los partidarios de lo que se conoce como teorías de los factores comunes. El punto de partida de esta escuela fue acertada y radicalmente planteado por Frank (1961). En realidad la postura sostenida 
por los partidarios de la integración teórica (explicar cómo pueden operar a la vez los factores terapéuticos hipotetizados por diferentes escuelas) parte de un prejuicio: El de suponer que las terapias basadas en cada una de las concepciones teóricas funcionan por los motivos por los que esa concepción supone que lo hacen. Si las terapias basadas en las diferentes orientaciones teóricas tienen resultados equivalentes, puede ser porque los factores hipotetizados por cada uno de ellas son efectivamente puestos en marcha mediante las técnicas diseñadas para ello, y tienen una potencia equivalente, como supondrían eclécticos o integradores teóricos. Pero también puede ser, como le parece a Frank, que lo que determina la eficacia de cada una de las intervenciones posibles, no es lo que tiene de específico y diferencial con las demás sino, precísamente, lo que comparte con ellas: por eso, porque lo que actúa es lo que todas tienen en común, el resultado es equivalente. En la hipótesis de Frank esos factores comunes son, además, los mismos que actúan en otras relaciones de ayuda, como las que establecen brujos o chamanes. Lo que en definitiva tienen en común, dice Frank, todas estas relaciones, es el hecho de estar estructuradas de tal modo que se dan en un encuadre determinado (el cromlech o el diván...), mediane un rito (el ensalmo o la asociación libre...), que está justificado por un mito en el que creen tanto ayudador como ayudado (mal de ojo o psicoaná- lisis). En esta línea de los factores comunes se incluyen los trabajos de Prochaska (Prochaska 1984; Prochaska y Di Clemente 1984), que ha desarrollado una teoría del cambio que permite planificar intervenciones considerando el momento del proceso de cambio a afrontar por el paciente, y los de Beitman (1987) que ha sistematizado los aconteceres de cualquier proceso psicoterapéutico en un sistema basado en un lenguaje común y que, recientemente, está trabajando en sofisticados sistemas de enseñanza del uso de los factores comunes en el proceso psicoterapéutico (Beitman, comunicación personal).

Definir los factores terapéuticos comunes de modo que den lugar a un sistema coherente, no es fácil y, por regla general, los intentos de ser exhaustivos se han acompañado de pérdidas en sistematicidad. Norcross y Arkowitz (1992) y Bergin y Garfield (1994) han recogido, bastante asístemáticamente por cierto, listados exhaustivos de factores de este tipo señalados por diversos autores. El cuadro 2 presenta el propuesto por el grupo de consenso al que antes hacíamos referencia (Fernández Liria, Hernández Monsalve, Rodríguez Vega, Benito Canoy Mas Hesse,1996) a partir de la reflexión sobre su práctica en el sector público madrileño.

El vuelco epistemológico derivado de la aplicación de las diferentes versiones del constructivismo que han invadido la teoría y la práctica de la psicoterapia, y la

Cuadro 2. Caracterización de una intervención psicoterapéutica: factores terapéuticos

1. Aprendizaje de nuevas conductas o estrategias de afrontamiento (habilidades sociales, relajacíon...)

2. Modificación o corrección de cogniciones acerca del mundo o uno mismo, o de mecanismos cognitivos

3. Reconocimiento o experiencia de emociones

4. Establecimiento de nexos entre emociones, experiencias internas y circunstancias externas

5. Experiencia emocional correctora en la relación con el terapeuta (incluye la aceptación empática)

6. Aprendizaje interpersonal (ponerse en el lugar del otro)

7. Sugestion

8. Modificación del entorno significativo del paciente (incluye reglas, estructuras, pautas disfuncionales...) 
consideración de práctica psicoterapéutica como una actividad narrativa, ofrece un lenguaje común para la consideración de los factores comunes y para la construcción de teorías integradoras, que ya ha sido aprovechado por muchos autores procedentes de distintas escuelas.

\section{A MODO DE CONCLUSIÓN}

Creo que lo hasta aquí dicho puede resumirse en algunas ideas muy simples que enuncio a continuación:

1) La psicoterapia es un procedimiento de tratamiento de los trastornos mentales y problemas de salud mental, avalado por la investigación empírica. Dado nuestro actual conocimiento sobre estos procedimientos, debe ser incluida, y de hecho lo está, entre las prestaciones ofertadas por el Sistema Nacional de Salud.

2) Las condiciones en las que la psicoterapia debe practicarse en el sector público (sin selección de pacientes, con la lexigencia de atender criterios de eficiencia y nó sólo de efectividad) determinan que, hoy por hoy, sea imposible entenderla en toda su extensión y practicarla de modo útil y coherente desde el marco de referencia exclusivo de ninguno de los modelos teóricos de que actualmente disponemos. Hay, pues, un especie de reclamo de las posiciones eclécticas o integradoras desde la práctica pública.

3) Tal reclamo permite presentar las tendencias clásicamente señaladas en el movimiento ecléctico integrador como respuestas a algunas de las necesidades básicas puestas de manifiesto por la práctica pública. Así,

a) La necesidad de disponer de un procedimiento sistemático para decidir qué intervención va a ser mas eficiente (no necesariamente aplicada por el mis- mo terapeuta que realiza la evaluación) para cada paciente, nos sitúa ante el tipo de problemas que tratan de resolver los autores del eclecticismo técnico.

b) La necesidad de proporcionar una formación básica y un lenguaje común para los profesionales que van a trabajar en el sector público, nos enfrenta a los problemas atendidos por los teóricos de los factores comunes. Estos autores nos proporcionan también el marco desde el que comprender los elementos psicoterapéuticos (o antiterapéuticos, o inútiles), que impregnan nuestra práctica cuando pensamos que sólo estamos realizando prescripción de fármacos o emitiendo juicios diagnósticos.

c) La necesidad de encuadrar nuestros conocimientos en un marco teórico general mas allá de lo que nos permiten las teorías sobre factores comunes, nos sitúa ante el tipo de problemas afrontados por los diversos intentos de integración teórica.

d) La óptica del constructivismo y el abandono de los últimos bastiones neopositivistas para enmarcar la psicoterapia en el campo de las narrativas, abren un camino para la construcción de un terreno de confluencia con un sistema y un lenguaje comunes que sin duda dará juego entre los terapeutas que trabajamos en el sector público.

\section{REFERENCIAS BIBLIOGRÁFICAS}

Alexander, F. (1963). The dynamics of psychotherapy in light of learning theory. American Journal of Psychiatry; 120, 440-448.

Beitman, B.D. (1987). The structure of individual psychotherapy. New York: Guilford Press,

Beutler, L.E. (1983). Eclectic psychotherapy: A systematic approach. New York: Pergamon.

Bergin, A.E., y Garfield, S.L. (1994). Handbook of psychotherapy and behavior change. New York: John Wiley \& Sons. 
Desviat, M., Fernández Liria, A., y Mas, J. (1996). Técnica y práctica psiquiátrica. En M. Desviat (Coord.), Epistemología y práctica psiquiátrica. Madrid: AEN.

Diéguez, M., Morales, N., y González, N. (1997). Terapia interpersonal de la depresión: un modelo de psicoterapia para el sector público. En A. Fernández Liria, M. Hernández y B. Rodríguez (Coords.), Psicoterapias en el sector público: un marco para la integración. Madrid: AEN.

Dollard, J. y Miller N.E. (1950). Personalidad y psicoterapia. Bilbao: Desclée de Brouwer, 1977

Eysenck, H.J. (1952). The effects of psychotherapy: An evaluation. Journal of Consulting Psychology, 16, 319-324.

Fairburn, C.G. (1993) Interpersonal psychotherapy for bulimia nervosa. En G.L. Klerman y M.M.Weissman (Eds.), New aplications of interpersonal psychotherapy. Washington: American Psychiatric Press.

Fairburn, C.G., Jones, R., Pevler, R.C., Carr, S.J., Solomon, R.A., O'Connor M.E., Burton, J., y Hope, R.A. (1991). Three psychological treatments for bulimia nervosa. Arch. Gen. Psychiatry, 48, 463-469.

Fernández Liria, A. (1993). Investigación en psicoterapia (I): el pájaro Dodo en el umbral del siglo XXI. Psiquiatría Pública, 5, 354-362.

Fernández Liria, A. (1994). Investigación en psicoterapia (II): especificidad e integración. Psiquiatría Pública, 6, 3-14.

Fernández Liria, A., Hernández, M., y Rodríguez, B. (Coords.) (1997). Psicoterapias en el sector público: un marco para la integración. Madrid: AEN.

Fernández Liria, A., Hernández, M., Rodríguez, B., Benito, T., y Mas, J. (1997b). Teoría y práctica de la psicoterapia en la atención pública a la salud mental: resultados de un grupo de consenso. En A. Fernández Liria, M. Hernández, y B. Rodríguez (Coords.), Psicoterapias en el sector público: un marco para la integración. Madrid: AEN.

Fernández Liria, A., y Rodríguez, B. (1997). La perspectiva integradora en psicoterapia: una reflexión desde la práctica pública. En A. Fernández Liria, M. Hernández y B. Rodríguez (Coords.), Psicoterapias en el sector público: un marco para la integración. Madrid: AEN.

Fernández Liria, A., Rodríguez, B., Diéguez, M., González, N., y Morales, N. (1997). La terapia interpersonal de la depresión y las psicoterapias en el fin del milenio. Revista de la Asociación Española de Neuropsiquiatria, 17, 627-648

Frank, J.D. (1961). Persuasion and Healing. Baltimore: John Hopkins University Press.

Garfield, S.L. (1994). Research on client variables in psychotherapy. En A.E. Bergin y S.L. Garfield, Handbook of psychotherapy and behavior change. IVth Edition (pp. 190-228). New York: Wiley.

Horowitz, M.J. (1988). Introduction to psychodinamics, a new synthesis. London: Routledge.

Horowitz, M.J. (1997). Formulation as a basis for planning psychotherapy treatment. Washington: American Psychiatric Press.

Horowitz, M.J., Marmar, C., y Krupnick, J. (1984). Personality styles and brief psychotherapy. New York: Basic Books.

Lambert M.J., y Bergin A.E. (1994). The effectiviness of psychotherapy. En A.E. Bergin y S.L. Garfield. Handbook of psychotherapy and behavior change. New York: Wiley.

Lazarus, A.A. (1981). The practice of multimodal therapy: systematic, compehensive and effective psychotherapy. New York: Mc-Graw Hill.

Lazarus A.A. (1992). Multimodal Therapy: Technical eclecticism with minimal integration. En J.C. Norcross y M.R.Goldfried (Eds.). Psychotherapy integration (pp. 231263). New York: Basic Books.

Luborsky, L., Singer, B., y Luborsky, L. (1975). Comparative studies of psychotherapy. Is it true that «everyone has won and all must have prizes"? Archives of General Psychiatry, 32, 995-1007.

Norcross, J.C. y Arkowitz, H. (1992). The evolution and current status of psychotherapy integration. En W. Dryden (Ed.), Integrative and eclectic therapy. A handbook. Buckingham: Plenum Press.

Prochaska, J.O. (1984). Systems of psychotherapy: a transtheoretical analysis. Homewood, Ill: Dorsey. 
Prochaska , J.O., y Di Clemente, C.C. (1984). The transtheoretical approach: crossing the traditional boundaries of therapy. Homewood: Dow Jones-Irrwin .

Rush, A.J. (1996). Assesing outcome in practice: a paradigm sjift? Current Opinion in Psychiatry, 9, 1-2.

Ryle, A. (1990). Cognitive-analytic therapy: active participation in change: a new integration in brief psychotherapy. Chichester; John Wiley.

Ryle, A. (Ed.) (1995a). Cognitive analytic therapy; developments in theory and practice. Chichester: Wiley.

Ryle, A. (1995b). The practice of CAT. En A.
Ryle (Ed.), Cognitive analytic therapy; developments in theory and practice. Chichester: Wiley.

Wachtel, P.L. (1977). Psychoanalysis and behaviour therapy; towards an integration. New York: Basic Books.

Wachtel, P.L. (1987). Action and insight. New York: Guilford.

Wachtel, P.L. (1993). Therapeutic Comunication; principles and effective practice. New York: Guilford.

Wachtel, P.L. (1997). Psychoanalysis, behaviour therapy; and the relational world. Washington: American Psychological Association. 